\title{
Relação entre o perfil antropométrico e bioquímico em crianças e adolescentes com diabetes melito tipo 1
}

Relationship between anthropometric and biochemical profiles in children and adolescents with type 1 diabetes

Sheylle Almeida S. Teles ${ }^{1}$, Nélida Schmid Fornés ${ }^{2}$

RESUMO

Objetivo: Avaliar a relação entre o perfil antropométrico e bioquímico de crianças e adolescentes com diabetes melito tipo 1 (DM1).

Métodos: Estudo transversal com 11 crianças e 43 adolescentes com DM1. Coletaram-se dados socioeconômicos e demográficos (idade, sexo, escolaridade, renda), clínicos (insulinoterapia), antropométricos (peso, estatura, dobras cutâneas, circunferência da cintura - CC) e bioquímicos (hemoglobina glicada - HbA, glicemias casual - GLC, pós-prandial - GLPP, e perfil lipídico). Foram utilizados o teste $t$ de Student $(p<0,05)$ e a correlação de Pearson $(p<0,05)$.

Resultados: A renda média per capita foi de 0,58 $\pm 0,39$ salário-mínimo e predominou o esquema de três aplicações de insulina/dia em $72,2 \%$ da amostra. A maioria apresentou estatura $(92,6 \%)$ e IMC (87\%) adequados para a idade. Aqueles com índice da $\mathrm{HbA}$ (inHbA) adequado apresentaram menores GLC $(p=0,002)$ e GLPP $(p<0,001)$. O inHbA correlacionou-se positivamente com CC $(p=0,013), \operatorname{GLC}(p=0,014), \operatorname{GLPP}(p<0,001)$, TG e VLDL $(p<0,001)$.

Conclusões: $\mathrm{O}$ pior controle glicêmico relaciona-se a maiores níveis de lipídeos séricos e CC mais elevada.

Palavras-chave: adolescente; antropometria; circunferência da cintura; criança; diabetes mellitus tipo 1; glicemia.

\section{ABSTRACT}

Objective: To evaluate the relationship between anthropometric and biochemical variables in children and adolescents with type 1 diabetes mellitus (DM1).

Methods: This was a cross-sectional study of 11 children and 43 adolescents with DM1. The following data were collected: socioeconomic and demographic (age, sex, education, income), clinical (insulin therapy), anthropometric (weight, height, skinfolds, waist circumference - WC) and biochemical variables (glycated hemoglobin - HbA, casual blood glucose - CBG, post-prandial blood glucose - PPBG, and lipid profile). Statistical analysis included Student's $t$ test $(p<0.05)$ and Pearson's correlation $(p<0.05)$.

Results: The average income per capita was $0.58 \pm 0.39$ times the monthly minimum wage and $72.2 \%$ of the sample were on insulin therapy consisting of three doses per day. Most individuals had adequate height (92.6\%) and BMI $(87.0 \%)$ for their ages. Subjects with an adequate $\mathrm{HbA}$ index (inHbA) had lower CBG $(p=0.002)$ and PPBG $(p<0.001)$. There were positive correlations between inHbA and $\mathrm{WC}$ $(p=0.013)$, CBG $(p=0.014)$, PPBG $(p<0.001)$, triglycerides and VLDL-cholesterol $(p<0.001)$.

Conclusions: Poorer glycemic control is related to higher serum lipids levels and larger WC.

Key-words: adolescent; anthropometry; waist circumference; child; diabetes mellitus, type 1; blood glucose.
Instituição: Ambulatório de Endocrinologia do Hospital das Clínicas da Universidade Federal de Goiás (UFG), Goiânia, GO, Brasil

${ }^{1}$ Mestre em Ciências da Saúde pela Faculdade de Medicina da UFG, Goiânia, GO, Brasil

2Pós-doutora em Medicina Preventiva e Saúde Pública pela Universidad Autónoma de Madri, Espanha; Professora da Faculdade de Nutrição da UFG, Goiânia, GO, Brasil
Endereço para correspondência:

Sheylle Almeida S. Teles

Rua I 3, quadra 05, lote 02 - Bairro Itamaraty, IV Etapa

CEP 75050-015 - Anápolis/GO

E-mail: sheyllealmeida@gmail.com

Fonte financiadora: Conselho Nacional de Desenvolvimento Científico e Tecnológico (CNPq) - bolsa de mestrado, processo n579392/2008-2 Conflito de interesse: nada a declarar

Recebido em: 19/4/2011

Aprovado em: 21/7/2011 


\section{Introdução}

O diabetes melito tipo 1 (DM1) é a endocrinopatia mais comum na infância e está associado ao aumento do risco cardiovascular $^{(1)}$. A hiperglicemia, a hipertensão e a dislipidemia atuam na gênese das complicações do $\mathrm{DM} 1^{(1)}$, acelerando diferentes fases da aterogênese ${ }^{(2)}$. As manifestações precoces das disfunções vasculares em indivíduos com DM1 são a rigidez e o espessamento das paredes arteriais ${ }^{(3,4)}$.

O Brasil está entre os dez países com maior número de casos de $\mathrm{DM}^{(5)}$. O país não possui um estudo de base populacional que identifique especificamente os casos de DM1. Estima-se que a prevalência e a incidência da doença em indivíduos menores de 14 anos sejam de 4/10.000 e 8/100.000 habitantes, respectivamente ${ }^{(6)}$.

O controle glicêmico intensivo (CGI) auxilia na prevenção das complicações, pois atua diretamente na redução dos níveis séricos de colesterol total (CT), lipoproteína de baixa densidade (LDL) e triglicérides (TG ${ }^{(7)}$. Por outro lado, ele promove o ganho ponderal e o aumento do percentual de gordura corporal (GC) em relação ao tratamento convencional ${ }^{(8)}$. O controle glicêmico inadequado, expresso por níveis elevados de hemoglobina glicada ( $\mathrm{HbA}$ ), está associado a maior tendência ao sobrepeso. Além disso, o número de aplicações de insulina foi identificado como preditor isolado e mais importante do índice de massa corporal (IMC) em indivíduos entre 12 e 17 anos $^{(9)}$.

Diante das evidências da relação entre controle metabólico e estado nutricional do portador de diabetes tipo 1 , objetivou-se avaliar a relação entre o perfil antropométrico e bioquímico em crianças e adolescentes com DM1.

\section{Método}

Estudo transversal envolvendo crianças e adolescentes portadores de DM1 atendidos no Ambulatório de Endocrinologia do Hospital das Clínicas da Universidade Federal de Goiás (UFG). No momento do estudo, a população com DM1 cadastrada no referido ambulatório era de 240 indivíduos, dos quais 31 eram crianças (12,0\%), 79 adolescentes $(32,2 \%)$ e 135 adultos $(55,8 \%)$. A amostra consensual foi constituída por $49 \%$ das crianças e adolescentes, totalizando 54 indivíduos voluntários.

O termo "criança" definiu indivíduos com idade entre quatro e nove anos e "adolescente" entre dez e 18 anos $^{(10)}$. Os critérios para inclusão no estudo foram: diagnóstico consolidado da doença há pelo menos cinco meses e estar em insulinoterapia. Os critérios de exclusão foram: amputação de membros, diagnóstico de dislipidemia, doença celíaca, nefropatia, tireoideopatias ou doenças cardiovasculares.

Os dados foram coletados no período de janeiro a agosto de 2009. O preenchimento dos critérios de inclusão e exclusão foi avaliado por meio de consulta ao prontuário. Foram convidados a participar do estudo todos os pacientes e responsáveis que estavam acompanhando as crianças e os adolescentes. Para a coleta de dados, foi utilizado um formulário estruturado abordando informações: a) Socioeconômicas e demográficas - idade, ocupação, escolaridade do voluntário, renda e estilo de vida; b) Avaliação antropométrica - peso, estatura, IMC, circunferência da cintura (CC), dobras cutâneas tricipital (DCT) e subescapular (DCSE); c) Exames laboratoriais - hemoglobina glicada (HbA), glicemias casual (GLC) e pós-prandial (GLPP), perfil lipídico, que inclui a dosagem de TG, $\mathrm{CT}_{\mathrm{s}}$, lipoproteína de alta densidade (HDL), lipoproteína de muito baixa densidade (VLDL), LDL. Os dados antropométricos foram aferidos de forma rotacional por um único avaliador. As medidas de peso, estatura e CC foram realizadas duas vezes, das dobras cutâneas, três vezes, obtendo-se, assim, as médias.

A estatura foi aferida por meio de estadiômetro portátil $\left(\right.$ Sanny $\left.{ }^{\circledR}\right)$ com $200 \mathrm{~cm}$ e divisões em milímetros. Para aferir o peso, foi utilizada balança plataforma digital $\left(\operatorname{Kratos}^{\circledR}\right)$, com capacidade máxima de 150 quilos e sensibilidade de 50 gramas. A CC foi medida com uma fita métrica inextensível. As dobras cutâneas foram obtidas com adipômetro (Lange ${ }^{\circledR}$ ).

Os dados de peso e estatura foram analisados e classificados por meio do software WHO Antro ${ }^{(11)}$, considerando os índices escore Z estatura/idade (Z E/I) e escore Z IMC/idade (Z IMC/I). A partir da classificação fornecida pelo software, a E/I foi considerada baixa quando $Z<-2$ e adequada quando $Z \geq-2$. Para o índice IMC/I, considerou-se magreza quando $Z<-2$, eutrofia quando $-2 \geq Z<+1$ e obesidade para $Z \geq+1^{(12)}$.

Para avaliar os dados de CC, foram utilizados os pontos de corte propostos por McCarthy, Jarrett e Crawley ${ }^{(13)}$.A DCT foi avaliada com base nos percentis propostos por Frisancho ${ }^{(14)}$.O protocolo de Slaughter et a ${ }^{(15)}$ foi utilizado para calcular a estimativa da composição corporal dos indivíduos entre oito e 18 anos. Como seis indivíduos tinham menos de oito anos de idade, não foi possível aplicar a equação. A partir dos valores de percentual de gordura, a amostra foi classificada segundo as categorias de adiposidade propostas por Lohman ${ }^{(16)}$, baseando-se na estratificação por sexo e categorias de adiposidade. Para 
meninos, considerou-se adiposidade baixa $<10 \%$, ótima entre 10 e $20 \%$, moderadamente alta e alta $>20 \%$; para meninas, considerou-se baixa $<15 \%$, ótima entre 15 e $25 \%$, moderadamente alta e alta $>25 \%$.Com a finalidade de tornar a apresentação dos dados mais clara, os indivíduos foram estratificados em "crianças" e "adolescentes" e agrupados nas categorias de adiposidade "baixa", "ótima" e "excessiva".

Os dados referentes ao perfil lipídico e HbA foram obtidos nos prontuários, coletando-se os mais recentes. Para os indivíduos cujo perfil lipídico havia sido realizado há mais de três meses, era solicitado um novo exame como parte da rotina de acompanhamento ambulatorial. Os exames de HbA eram trazidos a cada nova consulta, portanto os dados possuíam menos de duas semanas de coleta. Os dados de GLC eram coletados no acolhimento com aparelho de hemogluco teste (HGT) (Accu-chekAdvantage).

O perfil lipídico foi avaliado com base nos valores recomendados pela American Academy of Pediatrics ${ }^{(17)}$. Devido à variedade de metodologias empregadas para determinar os níveis de $\mathrm{HbA}$, adotou-se o índice da hemoglobina glicada (inHbA) para classificar o controle glicêmico (CG) em adequado e não adequado. $\mathrm{O}$ inHbA foi obtido dividindo-se o valor obtido da $\mathrm{HbA}$ pelo limite superior da normalidade do método, conforme Chase et a ${ }^{(18)}$. Assim, no presente trabalho, in $\mathrm{HbA}<1,33$ foi considerado CG adequado e inHbA $\geq 1,33$ CG, inadequado.

O estudo foi aprovado pelo Comitê de Ética em Pesquisa Médica Humana e Animal do Hospital das Clínicas da UFG. O termo de consentimento livre e esclarecido (TCLE) foi assinado voluntariamente por todos os participantes e seus responsáveis após os procedimentos envolvidos terem sido detalhadamente explicados.

O banco de dados foi elaborado em planilha do software Microsoft Excel (versão 2003) e analisado no Statistical Package for the Social Sciences (versão 17.0). A descrição socioeconômica e demográfica foi expressa em frequências e percentuais para as variáveis categóricas e medidas de tendência central, para as numéricas. A avaliação da distribuição das variáveis contínuas foi analisada pelo teste Kolmogorov-Smirnov (K-S), considerando-se distribuição normal valores de $p \geq 0,05$. A comparação entre os grupos com CG adequado e CG inadequado foi procedida por meio dos testes $t$ de Student $\mathrm{e}$ do qui-quadrado. O coeficiente de correlação de Pearson foi utilizado para avaliar a relação entre as variáveis bioquímicas e antropométricas.

\section{Resultados}

As características socioeconômicas, demográficas e antropométricas dos indivíduos estudados estão descritas na Tabela 1. Foram estudados, 11 crianças e 43 adolescentes, totalizando 54 indivíduos dos quais $29(54 \%)$ eram do sexo feminino e 25 (46\%) do masculino. Destaca-se que $83,3 \%$ tinham acima de nove anos de idade. A renda

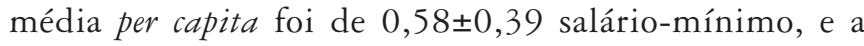
maioria das famílias era constituída por até quatro membros $(68,5 \%)$.

$\mathrm{O}$ esquema insulínico era composto de insulinas de ação intermediária (I) e rápida (R) em $66,7 \%$ dos casos,

Tabela 1 - Características socioeconômicas e demográficas das crianças e adolescentes com diabetes melito tipo 1

\begin{tabular}{|c|c|c|c|c|c|c|c|}
\hline & \multirow[t]{2}{*}{ Categorias } & \multicolumn{2}{|c|}{$\begin{array}{c}\text { Crianças } \\
(n=11)\end{array}$} & \multicolumn{2}{|c|}{$\begin{array}{l}\text { Adolescentes } \\
\quad(n=43)\end{array}$} & \multicolumn{2}{|c|}{$\begin{array}{l}\text { Total } \\
(n=54)\end{array}$} \\
\hline & & $\mathbf{n}$ & $\%$ & $\mathrm{n}$ & $\%$ & $\mathbf{n}$ & $\%$ \\
\hline \multirow[t]{2}{*}{ Sexo } & $\mathrm{F}$ & 6 & 54,5 & 23 & 53,5 & 29 & 53,7 \\
\hline & $M$ & 5 & 45,5 & 20 & 46,5 & 25 & 46,3 \\
\hline \multirow[t]{3}{*}{ Idade $\left(\right.$ anos) ${ }^{a}$} & $4-9$ & 11 & 100,0 & - & - & 11 & 20,4 \\
\hline & $10-13$ & - & - & 19 & 44,2 & 19 & 35,2 \\
\hline & $14-18$ & - & - & 24 & 55,8 & 24 & 44,4 \\
\hline Escolaridade (anos) ${ }^{\mathrm{b}}$ & & \multicolumn{2}{|c|}{$2,1 \pm 1,2$} & \multicolumn{2}{|c|}{$7,3 \pm 2,2$} & \multicolumn{2}{|c|}{-} \\
\hline Renda per capita (SM) b & & \multicolumn{2}{|c|}{$0,54 \pm 0,32$} & \multicolumn{2}{|c|}{$0,58 \pm 0,41$} & \multicolumn{2}{|c|}{$0,58 \pm 0,39$} \\
\hline \multirow{3}{*}{$\begin{array}{l}\text { Número de membros na } \\
\text { família }\end{array}$} & $2-3$ & 2 & 18,2 & 8 & 18,6 & 10 & 18,5 \\
\hline & 4 & 6 & 54,5 & 21 & 48,8 & 27 & 50,0 \\
\hline & $5-6$ & 3 & 27,3 & 14 & 32,6 & 17 & 31,5 \\
\hline \multirow[t]{2}{*}{ Atividade física } & $\operatorname{sim}$ & 4 & 36,4 & 31 & 72,1 & 35 & 64,8 \\
\hline & não & 7 & 63,6 & 12 & 27,9 & 19 & 35,2 \\
\hline
\end{tabular}

SM: salário mínimo; aMédia da idade \pm desvio padrão: $12,6 \pm 3,7 ;{ }^{b}$ média \pm desvio padrão 
Tabela 2 - Características clínicas e antropométricas das crianças e adolescentes com diabetes mellito tipo 1

\begin{tabular}{|c|c|c|c|c|c|c|c|}
\hline & \multirow[t]{2}{*}{ Categorias } & \multicolumn{2}{|c|}{$\begin{array}{c}\text { Crianças } \\
(n=11)\end{array}$} & \multicolumn{2}{|c|}{$\begin{array}{c}\text { Adolescentes } \\
\qquad(n=43)\end{array}$} & \multicolumn{2}{|c|}{$\begin{array}{c}\text { Total } \\
(n=54)\end{array}$} \\
\hline & & $n$ & $\%$ & $\mathbf{n}$ & $\%$ & $\mathbf{n}$ & $\%$ \\
\hline \multirow{4}{*}{ Esquema insulínico a } & $\mathrm{I} / \mathrm{R}$ & 5 & 45,5 & 31 & 72,1 & 36 & 66,7 \\
\hline & I/UR & 4 & 36,4 & 4 & 9,3 & 8 & 14,8 \\
\hline & LD/UR & 1 & 9,1 & 5 & 11,6 & 6 & 11,1 \\
\hline & LD ou I & 1 & 9,1 & 3 & 7,0 & 4 & 7,4 \\
\hline \multirow{2}{*}{$\begin{array}{l}\text { Número de aplicações de } \\
\text { insulina/dia }\end{array}$} & 3 & 8 & 72,7 & 31 & 72,1 & 39 & 72,2 \\
\hline & 4 & - & - & 6 & 14,0 & 6 & 11,1 \\
\hline \multirow{2}{*}{ Z E/I } & Baixa & - & - & 4 & 9,3 & 4 & 7,4 \\
\hline & Adequada & 11 & 100 & 39 & 90,7 & 50 & 92,6 \\
\hline Z IMC/I & Magreza & 1 & 9,1 & 2 & 4,7 & 3 & 5,6 \\
\hline $\mathrm{CC}$ & $>$ p90 & 3 & 27,3 & 11 & 25,6 & 14 & 25,9 \\
\hline \multirow{3}{*}{ DCT } & $<p 10$ & 1 & 9,1 & - & - & 1 & 1,9 \\
\hline & p10-p90 & 8 & 72,7 & 39 & 90,7 & 47 & 87,0 \\
\hline & $>$ p90 & 2 & 18,2 & 4 & 9,3 & 6 & 11,1 \\
\hline \multirow{4}{*}{ GC } & Baixa & 1 & 9,1 & 1 & 2,3 & 2 & 3,7 \\
\hline & Ótima & 3 & 27,3 & 22 & 51,2 & 25 & 46,3 \\
\hline & Excessivab $^{b}$ & 1 & 9,1 & 20 & 46,5 & 21 & 38,9 \\
\hline & $<8$ anos $^{c}$ & 6 & 54,5 & - & - & 6 & 11,1 \\
\hline
\end{tabular}

I: intermediária; R: rápida; UR: ultrarrápida; LD: longa duração.Z E/I: escore de desvios padrão de estatura para idade. Z IMC/I: escore de desvios padrão de índice de massa corporal para idade. CC: circunferência da cintura (avaliação em percentil) DCT: dobra cutânea triciptal; GC: gordura

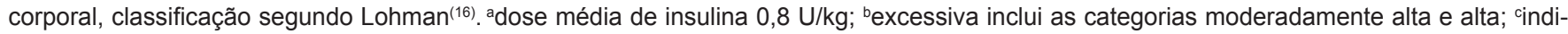
víduos para os quais não foi possível estimar o \%GC

Tabela 3 - Média e desvio padrão das características antropométricas e bioquímicas, segundo controle glicêmico

\begin{tabular}{|c|c|c|c|c|}
\hline & Valores de referência & CG adequado inHbA $<1,33$ & $C G$ inadequado inHbA $\geq 1,33$ & Valor $p$ \\
\hline Indivíduos (n/\%) & - & $35 / 65$ & $19 / 35$ & - \\
\hline Sexo M/F (n) & - & $14 / 21$ & $11 / 08$ & 0,208 \\
\hline Idade \pm DP (anos) & - & $12,3 \pm 3,9$ & $13,2 \pm 3,4$ & 0,400 \\
\hline Z E/I & $Z \geq-2$ & $-0,5 \pm 1,3$ & $0,0 \pm 1,0$ & 0,197 \\
\hline $\mathrm{Z} \mathrm{IMC} / \mathrm{I}$ & $-2 \geq Z \leq+1$ & $-0,4 \pm 1,2$ & $0,0 \pm 0,7$ & 0,179 \\
\hline \multirow[t]{2}{*}{ GC $(\%)$} & $\delta: \geq 10$ e $\leq 20$ & $15,8 \pm 6,2$ & $18,4 \pm 3,4$ & 0,250 \\
\hline & $q: \geq 15$ e $\leq 25$ & $25,7 \pm 8,7$ & $28,4 \pm 4,9$ & 0,441 \\
\hline GLC (mg/dL) & $<200$ & $227,8 \pm 117,3$ & $342,9 \pm 133,1$ & 0,002 \\
\hline GLPP (mg/dL) & $<140$ & $207,2 \pm 98,5$ & $338,0 \pm 111,5$ & $<0,001$ \\
\hline \multirow[t]{2}{*}{$\mathrm{TG}(\mathrm{mg} / \mathrm{dL})$} & $<10$ anos: $\leq 100$ & $78,6 \pm 26,9$ & $62,0 \pm 10,5$ & 0,326 \\
\hline & $10-19$ anos: $\leq 130$ & $83,5 \pm 33,9$ & $120,1 \pm 55,7$ & 0,028 \\
\hline $\mathrm{CT}_{\mathrm{s}}(\mathrm{mg} / \mathrm{dL})$ & $<200$ & $156,6 \pm 28,4$ & $163,4 \pm 29,5$ & 0,418 \\
\hline \multirow[t]{2}{*}{$\mathrm{HDL}(\mathrm{mg} / \mathrm{dL})$} & $<10$ anos: $\geq 40$ & $54,8 \pm 12,9$ & $38,0 \pm 8,1$ & 0,057 \\
\hline & $10-19$ anos: $\geq 35$ & $49,5 \pm 11,1$ & $44,9 \pm 7,9$ & 0,163 \\
\hline VLDL (mg/dL) & - & $16,1 \pm 6,4$ & $21,7 \pm 11,2$ & 0,054 \\
\hline $\mathrm{LDL}(\mathrm{mg} / \mathrm{dL})$ & $<130$ & $89,7 \pm 25,0$ & $98,0 \pm 24,6$ & 0,251 \\
\hline
\end{tabular}

Z E/I: escore de desvios padrão de estatura para idade; Z IMC/I: escore de desvios padrão de índice de massa corporal para idade; GC: gordura

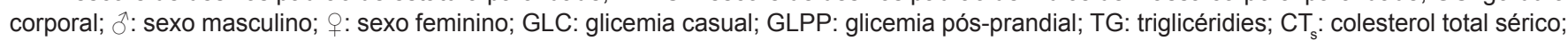
HDL: lipoproteína de alta densidade; VLDL: lipoproteína de muito baixa densidade; LDL: lipoproteína de baixa densidade 
Tabela 4 - Correlação entre variáveis antropométricas e bioquímicas em crianças e adolescentes com DM1

\begin{tabular}{lrccccccc}
\hline & \multicolumn{2}{c}{ inHbA } & \multicolumn{2}{c}{ LDL } & \multicolumn{2}{c}{ CT } & \multicolumn{2}{c}{ TG } \\
\cline { 2 - 9 } & \multicolumn{1}{c}{ Valor $\boldsymbol{p}$} & $\mathbf{r}$ & Valor $\boldsymbol{p}$ & $\mathbf{r}$ & Valor $\boldsymbol{p}$ & $\mathbf{r}$ & Valor $\boldsymbol{p}$ \\
\hline inHbA & 1,000 & - & 0,160 & 0,249 & - & - & - & - \\
CC $(\mathrm{cm})$ & 0,336 & $0,013^{*}$ & 0,030 & 0,830 & 0,134 & 0,335 & 0,313 & $0,021^{*}$ \\
DCT $(\mathrm{mm})$ & $-0,061$ & 0,664 & 0,066 & 0,633 & 0,114 & 0,413 & 0,017 & 0,902 \\
DCSE $(\mathrm{mm})$ & 0,175 & 0,206 & 0,061 & 0,661 & 0,153 & 0,271 & 0,239 & 0,082 \\
GC $(\%)$ & $-0,029$ & 0,844 & 0,069 & 0,639 & 0,124 & 0,403 & 0,046 & 0,754 \\
GLC $(\mathrm{mg} / \mathrm{dL})$ & 0,332 & $0,014^{*}$ & 0,075 & 0,587 & $-0,003$ & 0,982 & 0,128 & 0,356 \\
GLPP $(\mathrm{mg} / \mathrm{dL})$ & 0,490 & $<0,001^{* * *}$ & 0,092 & 0,507 & 0,009 & 0,949 & 0,196 & 0,155 \\
TG $(\mathrm{mg} / \mathrm{dL})$ & 0,422 & $0,001^{* *}$ & 0,149 & 0,283 & 0,478 & $<0,001^{* * *}$ & 1,000 & - \\
CT $(\mathrm{mg} / \mathrm{dL})$ & 0,201 & 0,146 & 0,854 & $<0,001^{* * *}$ & 1,000 & - & - & - \\
HDL $(\mathrm{mg} / \mathrm{dL})$ & $-0,196$ & 0,155 & $-0,091$ & 0,513 & 0,344 & $0,011^{*}$ & 0,142 & 0,307 \\
VLDL $(\mathrm{mg} / \mathrm{dL})$ & 0,443 & $0,001^{* *}$ & 0,093 & 0,503 & 0,431 & $0,001^{* *}$ & 0,975 & $<0,001^{* * *}$ \\
\hline
\end{tabular}

inHbA: índice da hemoglobina glicada; LDL: lipoproteína de baixa densidade; CT: colesterol total; TG: triglicérides; CC: circunferência da cintura; DCT: dobra cutânea triciptal; DCSE: dobra cutânea subescapular; GC: gordura corporal; GLC: glicemia casual; GLPP: glicemia pós-prandial; TG: triglicérides; $\mathrm{CT}_{\mathrm{s}}$ : colesterol total sérico; HDL: lipoproteína de alta densidade; VLDL: lipoproteína de muito baixa densidade; teste $T$ de Student: ${ }^{*} p<0,05 ;{ }^{* *} p<0,01 ;{ }^{* * *} p<0,001$

ao passo que o uso de análogos de ação longa (LD) e ultrarrápida (UR) ocorreu em 11,1\% (Tabela 2). A maioria dos indivíduos $(72,2 \%)$ realizava três aplicações de insulina ao dia. Apesar de $87 \%$ estarem eutróficos pelo Z-IMC/idade, 25,9\% apresentaram CC acima do percentil 90. Quanto à adiposidade, aproximadamente metade dos indivíduos $(46,3 \%)$ mostrou percentual de GC adequado, no entanto, 38,9\% tiveram adiposidade acima do recomendado.

Na Tabela 3, os dados antropométricos e bioquímicos estão estratificados segundo o CG, que se revelou inadequado em $35 \%$ dos indivíduos, sendo a maioria do sexo masculino (58\%). O grupo com CG inadequado mostrou níveis significantemente maiores de GLC (ambos os grupos apresentaram valores máximos acima de $500 \mathrm{mg} / \mathrm{dL}$ e GLPP (sendo $150 \mathrm{mg} / \mathrm{dL}$ o valor mínimo no grupo com CG inadequado). O nível de TG foi superior no grupo de indivíduos entre dez e 19 anos com CG inadequado $(p<0,05)$. Os níveis de HDL nos indivíduos abaixo de dez anos foram discretamente maiores no grupo com CG adequado, porém a diferença não foi significante $(p=0,057)$.

A avaliação da relação entre as variáveis é apresentada na Tabela 4. Houve correlação significativa entre o inHbA e as variáveis CC ( $\mathrm{r}=0,336 ; p=0,013)$, GLC ( $\mathrm{r}=0,332 ; p=0,014)$, GLPP ( $\mathrm{r}=0,49 ; p<0,001)$, TG ( $\mathrm{r}=0,422 ; p=0,001)$ e VLDL $(\mathrm{r}=0,443 ; p=0,001)$. Houve correlação significante do LDL com CT $(\mathrm{r}=0,85 ; p<0,001)$. O TG se correlacionou com CC $(\mathrm{r}=0,313 ; p=0,021)$ e VLDL $(\mathrm{r}=0,97 ; p<0,001)$.

\section{Discussão}

$\mathrm{O}$ inHbA apresentou correlação significativa com CC, GLC, GLPP, TG e VLDL, apontando que a glicemia e o perfil lipídico influenciam o controle metabólico de pacientes portadores de DM1. A relação entre hiperglicemia e desenvolvimento de complicações cardiovasculares foi demonstrada em um estudo experimental com camundongos portadores de diabetes. O grupo de camundongos que consumia uma dieta isenta de colesterol apresentou lesões ateroscleróticas significativamente maiores em comparação aos não diabéticos ${ }^{(2)}$.

Após estratificação pelo controle glicêmico, o grupo de pacientes com inHbA $\geq 1,33$ revelou maior risco cardiovascular, expresso pela correlação significativa com os níveis de GLC, GLPP, TG naqueles entre dez e 19 anos e de HDL, no limiar da significância, em indivíduos até dez anos. Apesar da relação entre controle glicêmico inadequado e dislipidemia ser um achado consistente na literatura, o mecanismo pelo qual a hiperglicemia se relaciona à aterosclerose ainda não foi completamente elucidado. Assim, a recomendação é de que, além do controle glicêmico, seja realizado o rigoroso monitoramento da pressão arterial e dos lipídeos séricos em portadores de DM1, a fim de evitar o desenvolvimento de complicações cardiovasculares ${ }^{(19)}$.

Este estudo não avaliou o déficit estatural, mas sabe-se que o DM1 está associado à redução na altura final dos indivíduos, comprometendo o crescimento em crianças e adolescentes ${ }^{(20,21)}$. 
O perfil antropométrico identificado neste trabalho é satisfatório, devido à maioria apresentar-se eutrófica, com adequada estatura para a idade no momento da avaliação.

Quanto à GC, considera-se preocupante que 38,9\% dos avaliados possuiam adiposidade acima do desejável. No entanto, é necessário considerar os indícios de que a porcentagem de massa gorda seja superestimada com medidas de dobras cutâneas, em comparação a avaliações efetuadas por raio X duo-energético (DEXA), especialmente em diabéticos com alto conteúdo de gordura. A glicação do colágeno no tecido subcutâneo pode contribuir para o endurecimento deste tecido e, assim como os achados precoces de redução de mobilidade articular, pode ser uma consequência prematura do DM1, antecedendo outras complicações em longo prazo $^{(22)}$.Por outro lado, um estudo que comparou diabéticos entre um e 11 anos a crianças não diabéticas não identificou diferença no percentual de gordura e massa magra ${ }^{(21)}$. Portanto, no presente estudo não foi possível verificar se o número de indivíduos com adiposidade acima do desejável é uma consequência do DM1 ou um fato inerente à infância e adolescência.

A CC é uma medida da obesidade central e reflete a gordura visceral metabolicamente ativa ${ }^{(23)}$. O inHbA foi significativamente correlacionado à CC, o que corrobora os achados do Diabetes Control and Complications Trial (DCCT) ${ }^{(24)}$. Diversos estudos apontam relação da CC com a rigidez vascular, estágio precoce da aterosclerose ${ }^{(25)}$, mortalidade cardiovascular ${ }^{(26)}$, desenvolvimento de microalbuminúria ${ }^{(24)}$ e surgimento da síndrome metabólica no DM1 ${ }^{(27)}$. Existem evidências da relação entre insulinoterapia, aumento da CC e ganho ponderal, especialmente naqueles em CG intensivo, sugerindo que o aumento da CC seja influenciado pela insulinoterapia ${ }^{(8)}$.

O nível elevado do inHbA denota controle metabólico ineficiente e foi correlacionado ao incremento na GLPP. Como consequência da hiperglicemia crônica, um estudo in vitro revelou maior glicação do LDL, tornando-o mais suscetível ao processo oxidativo ${ }^{(28)}$. Estes resultados reforçam o fato de que o CG inadequado aumenta o risco cardiovascular no DM1. Dados do Epidemiology of Diabetes Complications

\section{Referências bibliográficas}

1. Lachica LA, García MC. Diabetic nephropathy and cardiovascular risk. Aten Primaria 2010;42:173-5

2. Renard CB, Kramer F, Johansson F, Lamharzi N, Tannock LR, von Herrath MG et al. Diabetes and diabetes-associated lipid abnormalities have distinct effects on initiation and progression of atherosclerotic lesions. J Clin Invest 2004;114:659-68. apontam relação entre a $\mathrm{HbA}$ e o ganho de peso, sendo que os indivíduos com pior CG apresentaram maior ganho ponderal ${ }^{(29)}$. Neste estudo, houve correlação entre o Z IMC/I e inHbA, porém esta não foi significativa, possivelmente pelo reduzido tamanho da amostra.

Em jovens entre oito e 18 anos, portadores de DM1, observou-se que o aumento de IMC, CC, DCT, DCSE e GC elevou proporcionalmente a resistência insulínica. Maiores níveis de $\mathrm{HbA}$ também foram associados a esta resistência. A consequente incapacidade de captar glicose foi relacionada ao aumento nas concentrações de $\mathrm{CT}_{\mathrm{s}}$, $\mathrm{LDL}$ e TG e diminuição dos níveis de $\mathrm{HDL}^{(30)}$.

O desenho da pesquisa não permite avaliar o fator causal da relação entre parâmetros bioquímicos e antropométricos. No entanto, os resultados reforçam a importância do controle glicêmico para reduzir o risco cardiovascular no DM1. O número de indivíduos incluídos na amostra foi um fator limitante, porém isto decorreu da tentativa de compreender a relação entre o perfil antropométricos e bioquímico de portadores de DM1 sem complicações da doença. A inclusão de indivíduos que apresentassem doenças associadas poderia influenciar os resultados, especialmente quanto aos lipídeos séricos.

Em síntese, o estudo revelou que o CG inadequado está associado a maiores níveis de lipídeos séricos, especificamente TG e VLDL, o que denota maior risco cardiovascular. A correlação entre CC e inHbA aponta a importância de incluir a medida da CC na rotina de atendimento ambulatorial de jovens com DM1, permitindo o rastreamento de indivíduos com risco cardiovascular. Para isto, é necessário desenvolver pontos de corte de CC específicos para este grupo. Além disso, o monitoramento dos níveis de lipídeos séricos é essencial para alcançar e manter o controle metabólico adequado.

\section{Agradecimentos}

Às professoras Maria Luiza Ferreira Stringhini e Rosana de Moraes Borges Marques pelo auxílio na condução do estudo.

3. Rönnback M, Fagerudd J, Forsblom C, Pettersson-Fernholm K, Reunanen A et al. Altered age-related blood pressure pattern in type 1 diabetes. Circulation 2004;110:1076-82.

4. Giannattasio C, Failla M, Piperno A, Grappiolo A, Gamba P, Paleari F et al. Early impairment of large artery structure and function in type I diabetes mellitus. Diabetologia 1999;42:987-94 
5. Wild S, Roglic G, Green A, Sicree R, King H. Global prevalence of diabetes: estimates for the year 2000 and projections for 2030. Diabetes Care 2004;27:1047-53.

6. Collado-Mesa F, Barceló A, Arheart KL, Messiah SE. An ecological analysis of childhood-onset type 1 diabetes incidence and prevalence in Latin America. Rev PanamSaludPublica 2004;15:388-94.

7. No authors listed. Effect of intensive diabetes management on macrovascular events and risk factors in the Diabetes Control and Complications Trial. Am J Cardiol 1995;75:894-903.

8. No authors listed. Influence of intensive diabetes treatment on body weight and composition of adults with type 1 diabetes in the Diabetes Control and Complications Trial. Diabetes Care 2001;24:1711-21.

9. Holl RW, Grabert M, Sorgo W, Heinze E, Debatin KM. Contributions of age, gender and insulin administration to weight gain in subjects with IDDM. Diabetologia 1998;41:542-7.

10. Organização Pan-Americana de Saúde. La salud del adolescente y el joven en las Américas. Washington: OPS; 1985.

11. WorldHealth Organization. WHO Anthro for personal computers manual: software for assessing growth and development of the world's children. Geneva: WHO; 2010.

12. Brasil. Ministério da Saúde. Secretaria de Atenção à Saúde - Departamento de Atenção Básica. Protocolos do Sistema de Vigilância Alimentar e Nutricional - SISVAN na assistência à saúde. Brasília: Ministério da Saúde, 2008.

13. McCarthy HD, Jarrett KV, Crawley HF. The development of waist circumference percentiles in British children aged 5.0-16.9 y. Eur J ClinNutr 2001;55:902-7.

14. Frisancho AR. New norms of upper limb fat and muscle areas for assessment of nutritional status. Am J ClinNutr 1981;34:2540-5.

15. Slaughter MH, Lohman TG, Boileau RA, Horswill CA, Stillman RJ, Van Loan $\mathrm{MD}$ et al. Skinfold equations for estimation of body fatness in children and youth. Hum Biol 1988;60:709-23.

16. Lohman TG. The use of skinfolds to estimate body fatness on children and youth. JOPERD 1987;58:98-102.

17. No authors listed. National Cholesterol Education Program (NCEP): highlights of the report of the expert panel on blood cholesterol levels in children and adolescents. Pediatrics 1992;89:495-501.

18. Chase HP, Jackson WE, Hoops SL, Cockerman RS, Archer PG, O'Brien D. Glucose control and the renal and retinal complications of insulin-dependent diabetes. JAMA 1989;261:1155-60.
19. Conget I, Giménez M. Glucose control and cardiovascular disease: is it important? No. Diabetes Care 2009;32 (Suppl 2):S334-6.

20. Meira SO, Morcillo AM, Lemos-Marini SH, Paulino MF, Minicucci WJ, GuerraJúnior G. Pubertal growth and final height in 40 patients with type 1 diabetes mellitus. Arq Bras Endocrinol Metab 2005;49:396-402.

21. Paulino MF, Lemos-Marini SH, Guerra-Júnior G, Minicucci WJ, Mendes CT, Morcillo AM. Growth and body composition in children with type 1 diabetes mellitus. Arq Bras Endocrinol Metab 2006;50:490-8.

22. Ingberg CM, Särnblad S, Palmér M, Schvarcz E, Berne C, Aman J. Body composition in adolescent girls with type 1 diabetes. Diabet Med 2003;20:1005-11.

23. Nieves DJ, Cnop M, Retzlaff B, Walden CE, Brunzell JD, Knopp RH et al. The atherogenic lipoprotein profile associated with obesity and insulin resistance is largely attributable to intra-abdominal fat. Diabetes 2003;52:172-9.

24. De Boer IH, Sibley SD, Kestenbaum B, Sampson JN, Young B, Cleary PA et al Central obesity, incident microalbuminuria, and change in creatinine clearance in the epidemiology of diabetes interventions and complications study. J Am Soc Nephrol 2007;18:235-43.

25. Wadwa RP, Urbina EM, Anderson AM, Hamman RF, Dolan LM, Rodriguez $\mathrm{BL}$ et al. Measures of arterial stiffness in youth with type 1 and type 2 diabetes: the SEARCH for diabetes in youth study. Diabetes Care 2010;33:881-6.

26. Conway B, Miller RG, Costacou T, Fried L, Kelsey S, Evans RW et al. Adiposity and mortality in type 1 diabetes. Int J Obes (Lond) 2009;33:796-805.

27. Nádas J, Putz Z, Fövényi J, Gaál Z, Gyimesi A, Hídvégi T et al. Cardiovascular risk factors characteristic for the metabolic syndrome in adult patients with type 1 diabetes. Exp Clin Endocrinol Diabetes 2009;117:107-12.

28. De Castro SH, Castro-Faria-Neto HC, Gomes MB. Association of postprandial hyperglycemia with in vitro LDL oxidation in non-smoking patients with type 1 diabetes - a cross-sectional study. Rev Diabet Stud 2005;2:157-64.

29. Williams KV, Erbey JR, Becker D, Orchard TJ. Improved glycemic control reduces the impact of weight gain on cardiovascular risk factors in type 1 diabetes. The Epidemiology of Diabetes Complications Study. Diabetes Care 1999;22:1084-91.

30. Szadkowska A, Pietrzak I, Mianowska B, Bodalska-Lipińska J, Keenan HA, Toporowska-Kowalska E et al. Insulin sensitivity in type 1 diabetic children and adolescents. Diabet Med 2008;25:282-8. 